\title{
Recuperação da informação em psicologia: LILACS e Index Psi Revistas Técnico-Científicas
}

\author{
Elaine Cristina Domingues Martins
}

\begin{abstract}
Bibliotecária da seção de tratamento da informação da Biblioteca Dante Moreira Leite do Instituto de Psicologia da USP
\end{abstract}

Tatiana Carvalho

Técnica Administrativa da seção de tratamento da informação da Biblioteca Dante Moreira Leite do Instituto de Psicologia da USP.Mestre em Educação pela Faculdade de Educação da Universidade de São Paulo

http://dx.doi.org/10.1590/1981-5344/1615

Este artigo questiona se a recuperação da informação da área de psicologia, na base LILACS (Literatura LatinoAmericana e do Caribe em Ciências da Saúde), fica comprometida, quando não é utilizada uma terminologia específica. Analisa-se a indexação de 124 artigos de periódicos de psicologia, que compõem as bases de dados LILACS e Index Psi Revistas Técnico-Científicas (Base de dados que contribui para o controle bibliográfico da literatura brasileira em psicologia publicada em periódicos). Observa-se que $52 \%$ dos artigos foram bem representados pela Terminologia em Psicologia por termos que especificam mais os conceitos da área; 35\% foram representados idêntica ou similarmente pelos dois vocabulários; e $13 \%$ foram melhores indexados, utilizando-se os DeCS (Descritores em Ciências da Saúde). Conclui-se que uma possível solução para realizar uma boa representação da área de psicologia seria a inclusão de mais termos no DeCS, já que essa área contribui com 78 títulos de revistas especializadas na LILACS, correspondendo, até o momento, a 16.324 artigos cadastrados.

Palavras-chave: Recuperação da informação; Indexação; Terminologias; Revistas científicas. 


\section{Information retrieval in Psychology: LILACS and Index Psi Revistas Técnico-Científicas}

This article questions if information retrieval of Psychology on the Lilacs database is affected when a specific terminology is not used. Indexing of 124 journal articles in psychology that comprises the databases LILACS (Latin-American and Caribbean Center on Health Sciencies Information) and Index Psi is analyzed. We observed that $52 \%$ of the articles were well represented by the terminology in psychology, for more specific terms of the area; $35 \%$ were similarly represented by both vocabularies, and $13 \%$ were better indexed by using DeCS (Descriptors in Health Sciences, developed from $\mathrm{MeSH}$ ). We conclude that a possible solution to achieve a good representation of Psychology would be the inclusion of more terms in the DeCS, because this area contributes with 78 titles of journals in LILACS corresponding until now to 16.324 registered articles.

Keywords: Information retrieval; Indexing; Terminology; Scientific Journals.

Recebido em 03.10.2012 Aceito em 12.02.2014

\section{Introdução}

As Linguagens documentárias auxiliam os indexadores a escolherem os termos apropriados para representar o conteúdo de um documento.

Boccato e Fujita (2011) nos ajudam a entender a importância das Linguagens Documentárias:

Os vocabulários controlados são instrumentos de representação temática que possibilitam organizar a informação documentária para recuperação por assunto, a partir de estratégias de busca elaboradas por usuários locais e a distância (BOCCATO; FUJITA, 2011, p. 1).

A função principal de um vocabulário controlado é organizar a informação especializada e o da indexação é traduzir a linguagem natural do pesquisador em linguagem documentária, por meio da representação do conceito. Essa representação é útil para que os pesquisadores se apropriem de outros conhecimentos anteriormente gerados e possam 
produzir mais ciência, fortalecendo sua área de atuação. Dinamizando essa ponte entre as pesquisas e os pesquisadores, estão as bases de dados, especializadas em suas respectivas áreas, que, por meio de buscas, apresentam o conteúdo atualizado e apropriado, o que contribui para a evolução da ciência. Portanto, quando o documento é bem representado tematicamente, o pesquisador e sua área são beneficiados.

Há maior possibilidade de recuperação de um trabalho científico, de ser lido e citado, quando ele é bem representado. Manhã et al. (2008) explicam:

A visibilidade dos artigos leva à citação. A citação é o fator motivador para o pesquisador publicar. É um indicador importante para a avaliação da capacidade científica tanto do indivíduo, quanto departamento, universidade ou país. É importante para a sobrevivência da própria revista citada (MANHÃ et al., 2008, p. 2).

O trabalho do indexador é primordial para que a disseminação da informação possa acontecer de forma mais eficiente possível. As bases de dados são instrumentos igualmente importantes e essenciais para a evolução da ciência, divulgando as informações para toda a comunidade por meio dos artigos das revistas especializadas.

Esta relação pode ser traduzida da seguinte maneira: parceria do pesquisador + indexador + base de dados $=0$ desenvolvimento $\mathrm{e}$ divulgação do conhecimento científico das áreas especializadas, enriquecendo e oxigenando a ciência.

As revistas de Psicologia vêm obtendo aceitação em muitas bases de dados, como nos mostra Sampaio e Sabadini (2012). Uma das principais bases de dados brasileira, que incluem as revistas da área, é a Literatura Latino-americana e do Caribe em Ciências da Saúde (LILACS). As revistas de psicologia incluídas na base LILACS são cadastradas pelos bibliotecários dos Centros Cooperantes da Rede Brasileira de Bibliotecas da Área de Psicologia (ReBAP), que conta com 178 bibliotecas participantes, sendo Instituições de Ensino Superior Federais, Estaduais, Particulares, Associações e Sociedades de Psicologia. Vale ressaltar que esses colaboradores são pessoas comprometidas e envolvidas com 0 trabalho e que prestam esse serviço social à comunidade psi, sendo esta cooperação uma característica marcante do profissional bibliotecário. A maioria desses profissionais não trabalha em Instituições especializadas em Psicologia, mas em locais multidisciplinares, o que pode acarretar em uma indexação mais geral de documentos que são específicos de psicologia, por desconhecimento da área.

A ReBAP, que contribui para a manutenção das principais bases de dados que sustentam a Biblioteca Virtual em Saúde Psicologia Brasil (BVSPsi), utiliza a metodologia LILACS (Lildbi-WebPsi). Esta metodologia foi customizada pela Bireme, para cadastrar, de forma descentralizada, o conteúdo informacional dos materiais de psicologia. No caso dos 
periódicos, em uma única interface, alimentam-se três bases de dados: a base "mãe" é o Index Psi Revistas Técnico-Científicas, que conta hoje com 38.272 artigos. Dessa base são retirados extratos para as bases Index Psi Divulgação Científica e LILACS. Essas bases contêm dados referenciais e apontam para textos completos, quando disponíveis em acesso aberto. Para os títulos LILACS, devem-se indexar os artigos pelas duas terminologias (Descritores em Ciências da Saúde - DeCS - e Terminologia em Psicologia, ambas disponíveis online). Nesse momento, surge uma grande dificuldade na indexação dos materiais, pois, apesar da psicologia fazer parte da saúde mental, o DeCS não contempla a área com termos mais específicos.

A LILACS é altamente representativa no meio acadêmico para divulgação científica, tanto para os autores e pesquisadores como para os cientistas. Seu alcance ultrapassa fronteiras e fortalece ainda mais a área das ciências da saúde na América Latina e Caribe.

A LILACS é o mais importante e abrangente índice da literatura científica e técnica da América Latina e Caribe. Há 26 anos contribuindo para o aumento da visibilidade, acesso e qualidade da informação em saúde na Região ${ }^{1}$.

Portanto, aumentar a representação temática da Psicologia no DeCS é de grande valia para a área das Ciências da Saúde e da Saúde Mental especificamente, pois os textos de Psicologia ficam ocultos, quando representados por descritores muito gerais.

Lancaster (2004) aborda a seguinte questão:

O indexador especialista do assunto nem sempre é o que de fato indexa nas bibliotecas universitárias e a exigência de se o indexar determinada quantidade de documentos não permite que o indexador faça uma leitura necessária do documento, o que se sugere que ele somente faça um misto de leitura e 'passagem dos olhos' pelo texto (LANCASTER, 2004, p. 24).

Não se pode contestar a qualidade da indexação, por se tratar de um processo subjetivo, mas, por outro lado, ele pode interferir na eficácia da busca nas bases de dados pelos pesquisadores. Um documento deve ser bem analisado e representado para que os usuários finais possam se apropriar desses conhecimentos, continuando a produzir informações científicas. Para Arrimar (2010), trata-se de uma regra básica para realizar uma boa indexação.

Para a realização do trabalho complexo de indexação, é imperioso lembrar que o conhecimento tácito, o intrínseco, a experiência pessoal e profissional do indexador são muito relevantes para que o documento seja bem representado nas bases de dados.

${ }^{1}$ Disponível em: <http://lilacs.bvsalud.org/>. Acesso em: 2 set. 2012. 
A análise conceitual é a atividade de definição dos assuntos que são tratados no documento e a determinação dos termos de indexação corresponde à atividade de conversão dos conceitos identificados na análise para uma linguagem de indexação (ARRIMAR, 2010).

E completa a ideia em outro momento quando diz:

A coerência da indexação consiste na concordância quanto aos termos a serem usados para indexar um assunto tratado em diferentes documentos. O conceito de coerência é um dos requisitos para uma indexação de qualidade, pois todos os documentos que tratam o mesmo assunto devem estar representados da mesma forma, não devendo subsistir perda de informações no momento da recuperação (ARRIMAR, 2010, p. 7).

Uma terminologia especializada traz subsídios ao indexador para representar com maior precisão os documentos que estarão disponíveis em bases de dados para a comunidade científica realizar suas buscas.

A Psicologia no Brasil é uma área forte e organizada e é representada por uma Biblioteca Virtual especializada na área: BVS-Psi, coordenada pelo Instituto de Psicologia da USP, Conselho Federal de Psicologia e várias outras entidades significativas. Seu conteúdo tecnológico é gerido pela Bireme e seus produtos são desenvolvidos na modelagem estabelecida por eles. Portanto, todo o conteúdo informacional deve obrigatoriamente ser representado pelo seu sistema indexador - o DeCS. A utilização da metodologia LILACS, que permite com exclusividade a representação temática somente pelo DeCS, deve ser repensada, como mostraremos a seguir. Os colaboradores da ReBAP argumentam que não encontram os termos ideais para representar os conteúdos dos documentos, comprometendo, assim, o diálogo do usuário com o produto LILACS.

O objetivo do sistema de informação da BVS-Psi e da ReBAP é atender aos pesquisadores, indicando o que existe de mais especializado na literatura brasileira da área de psicologia. Contudo, há uma lacuna em relação à terminologia da área das ciências da saúde, por esta não representar tão bem quanto almejado o conteúdo temático da categoria psi.

A incorporação do repertório temático da Psicologia no DeCS se faz necessária por conta da grande dimensão que a área está alcançando, dada à visibilidade da Biblioteca Virtual. Packer (2011) confirma a importância da BVS, que evoluiu e consolidou-se como referência da informação científica e técnica da Psicologia:

A BVS-Psi representa avanço notável para a psicologia brasileira e a latino-americana, ao contribuir (de fato equacional), de modo eficiente, eficaz e contemporâneo, a indexação, a organização, a publicação e o acesso à informação científica e técnica, particularmente, a que é 
gerada e publicada nacionalmente. Esse equacionamento, que inclui o tratamento multilíngue, constitui uma das dimensões essenciais para o avanço da pesquisa, da educação e da prática profissional da Psicologia (PACKER, 2011, p. 43).

A Psicologia, por ser uma área altamente colaborativa com a Ciência da Saúde Mental, demanda que seus conceitos sejam bem representados pela terminologia oficial da LILACS, permitindo que os pesquisadores encontrem os documentos que foram indexados por meio dessa linguagem documentária - DeCS.

As terminologias devem ser mantidas sempre atualizadas e abertas para incorporação de novos descritores, atendendo ao processo natural do desenvolvimento da ciência. Borba, Van Der Laan e Chini (2012) esclarecem que:

O que se espera dessas linguagens é uma operacionalidade que integre o usuário e o sistema de informação dentro de um contexto informacional constituído por demandas informacionais de uma determinada área do conhecimento (BORBA; VAN DER LAAN; CHINI, 2012, p. 30).

A ReBAP participa com 78 títulos de periódicos de Psicologia no trabalho descentralizado de alimentação, manutenção e atualização da base LILACS, sendo 65 títulos correntes, até a data desta pesquisa, correspondendo a 16.324 artigos cadastrados. A fonte de consulta foi a Lista dos Periódicos Indexados que consta na Base Index Psi Revistas Técnico-Científicas, que contém 191 títulos, sendo 118 correntes $^{2}$.

\section{Breve apresentação das Terminologias utilizadas para o estudo}

\subsection{Terminologia em Psicologia}

A terminologia em Psicologia vem sendo construída, desde 1973, pela Biblioteca "Dante Moreira", do Instituto de Psicologia da USP, e atualmente conta com 6.433 termos. Ela se encontra disponível por meio do site da Biblioteca Virtual de Psicologia ULAPSI Brasil (BVS-PSi) ${ }^{3}$.

O passo inicial para elaboração dessa Terminologia foi a de compilar os termos extraídos da literatura e representá-los por meio dos conceitos na área da Psicologia. A Terminologia passa, hoje, por um processo de atualização e está na fase de tradução para o Inglês e Espanhol dos termos e, também, das Notas de Escopo, além da criação das mesmas para os que não a possuem. Em 1974, a American Psychological Association editou o Thesaurus of Psychological Index Terms, o que validou os termos anteriormente selecionados. Manteve-se a atualização

\footnotetext{
${ }^{2}$ Disponível em: <http://newpsi.bvs-psi.org.br/cgi- bin/wxis1660.exe/iah/?/sisScript=iah /iah.xis\&lang=P\&base=INDEXPSI> . Acesso em: 24 ago. 2012.

${ }^{3}$ Disponível em: < http://www.bvs-psi.org.br>. Acesso em: 24 ago. 2012.
} 
dessa obra e a consulta aos termos para a manutenção e aprimoramento da Terminologia em Psicologia, bem como de outros Tesauros específicos da área. Uma característica marcante da Terminologia em Psicologia é que os autores são incluídos como assuntos.

A Terminologia em Psicologia é o instrumento principal para a indexação dos materiais disponíveis na BVS-Psi Brasil e, também, para alguns países da América Latina ${ }^{4}$.

\subsection{Descritores em Ciências da Saúde (DeCS)}

O DeCS é o fundamental instrumento de indexação para a área das Ciências da Saúde e foi desenvolvido pela Bireme, a partir do Medical Subject Headings (MeSH ) da U.S. National Library of Medicine (NLM).

Seu maior objetivo é servir como uma linguagem única para indexar todos os materiais disponíveis na Biblioteca Virtual em Saúde (BVS), como LILACS, MEDLINE e outras. Conta, hoje, com 30.895 descritores, sendo destes 26.664 do MeSH e 4658 exclusivamente do DeCS. Segundo o site do DeCS:

Os conceitos que compõem o DeCS são organizados em uma estrutura hierárquica permitindo a execução de pesquisa em termos mais amplos ou mais específicos ou todos os termos que pertençam a uma mesma estrutura hierárquica. Além dos termos médicos originais do MeSH foram desenvolvidas as áreas específicas de Saúde Pública, Homeopatia, Ciência e Saúde e Vigilância Sanitária ${ }^{5}$.

A seguir, apresenta-se a avaliação das terminologias utilizadas para indexar os artigos de revistas na base LILACS e Index Psi Revistas TécnicoCientíficas.

\section{Metodologia}

A primeira etapa do trabalho foi a de recuperar os 78 títulos de periódicos de Psicologia da base LILACS, publicados entre os anos de 2010 e 2011. Destes, foram selecionadas 65 revistas, que são consideradas correntes, sendo, em seguida, numeradas de 1 a 65 para realização do sorteio de seis títulos. Escolheu-se trabalhar com $10 \%$ do número total das publicações, por elas oferecem um número relevante de artigos a serem analisados. Em seguida, foi solicitado a seis pessoas, que não tiveram contato com o teor da pesquisa, que escolhessem um número dentro desse universo.

Selecionadas as revistas, foram escolhidos os seis primeiros artigos de cada uma, de cada ano, número também considerado satisfatório.

\footnotetext{
4 Disponível em: <www.psi.homolog.bvs.br/php/level.php?lang=pt\&component=19\&item=95>. Acesso em: 24 ago. 2012.

${ }^{5}$ Disponível em: <http://decs.bvs.br/P/decsweb2012.htm>. Acesso em: 24 ago. 2012.
} 
Foram escolhidos os seis primeiros, justificando a variação da quantidade de artigos que ocorre nas revistas, totalizando 124 documentos.

Nem todos os títulos que constavam como correntes estavam, de fato, atualizados e, por isso, o número de artigos escolhidos oscilou. Apesar disso, as revistas selecionadas foram mantidas, demonstrando a falha na alimentação da base de dados, porém, não afetando os resultados da pesquisa.

A etapa seguinte foi elaborar uma planilha em Excel com os seguintes campos: revista, volume, número, ano, assuntos DeCS, assuntos Terminologia em Psicologia, demonstrando as indexações dos artigos realizadas pelos bibliotecários da ReBAP.

Por último, analisou-se se há comprometimento na recuperação da informação em psicologia, quando a indexação é realizada somente pela terminologia da área da saúde.

A busca foi realizada no site www.bvs-psi.org.br, em 24/08/2012, selecionando-se a base Index Psi Revistas Técnico-Científicas, que contém indexação pelas duas terminologias, e o campo "Revista", escolhendo posteriormente os anos correspondentes com a amostra da pesquisa. Foram apresentados os registros completos, inclusive com os dados das indexações.

A seguir, selecionaram-se os seis artigos de cada fascículo.

As revistas sorteadas e seus respectivos dados são apontados a seguir:

1)Curinga - Universidade Federal de Minas Gerais - 30-31, 2010 - Total= 35 artigos, sendo 12 utilizados para a pesquisa;

2)Mudanças: Psicologia da Saúde - Universidade Metodista de São Paulo 18(1/2), 2010 - Total= 10 artigos, sendo seis utilizados para a pesquisa;

3)Psico - Pontifícia Universidade Católica do Rio Grande do Sul 41(1-4), 42 (1-3), 2010 - Total= 108 artigos, sendo 40 utilizados para a pesquisa;

4)Revista Mal-Estar e Subjetividade - Universidade de Fortaleza 10 (1-4), 2010 - Total= 58 artigos, sendo 24 utilizados para a pesquisa;

5)Vínculo: Revista do NESME - Universidade de São Paulo Ribeirão Preto 8(1), 2011 - Total= 7 artigos, sendo seis utilizados para a pesquisa. O primeiro artigo foi descartado por ser o editorial e não apresentar dados de indexação; e

6)Estudos de Psicologia (Natal) - Universidade Federal do Rio Grande do Norte 15-16(1-3), 2010 - Total=85 artigos, sendo 36 utilizados para a pesquisa. Este título está corrente. O primeiro artigo é o editorial, e foi decidido não incorporá-lo ao escopo da pesquisa. 


\section{Resultados}

Foram analisados 124 artigos de revistas indexadas nas bases LILACS e Index Psi Revistas Técnico-Científicas, utilizando-se as terminologias específicas de cada base.

As duas terminologias foram satisfatórias para representar 0 conteúdo temático de 43 artigos, isto é, conseguiram contemplar a complexidade dos documentos, apresentando indexações com conceitos idênticos ou similares.

Por exemplo, o segundo artigo do Quadro 1, que fala sobre bemestar no trabalho, foi indexado com termos semelhantes pelas duas terminologias ("satisfação no emprego" e "satisfação no trabalho"), possibilitando sua recuperação. Neste mesmo caso, a palavra indexada pelo DeCS, "Poder", foi qualificada pela palavra "Psicologia", deixando o termo mais específico. E a indexação da mesma palavra, "Poder", pela Terminologia em Psicologia, não precisou ter o mesmo qualificador, por já representar o conteúdo da área.

Esse grupo correspondeu a aproximadamente $35 \%$ do total de artigos analisados.

Os exemplos estão apresentados no quadro a seguir:

Quadro 1 - Indexações com conceitos idênticos ou similares dos artigos das revistas de psicologia da base LILACS entre os anos 2010-2011

\begin{tabular}{|c|c|c|}
\hline Título do artigo & Indexação pelo DeCS & $\begin{array}{l}\text { Indexação pela Terminologia em } \\
\text { Psicologia }\end{array}$ \\
\hline $\begin{array}{l}\text { 1) O que há de constante no } \\
\text { autismo? }\end{array}$ & $\begin{array}{l}\text { Psicanálise } \\
\text { Transtorno autístico }\end{array}$ & $\begin{array}{l}\text { Psicanálise } \\
\text { Autismo }\end{array}$ \\
\hline $\begin{array}{l}\text { 2) Bem-estar no trabalho: influência } \\
\text { das bases de poder do supervisor e } \\
\text { dos tipos de conflito }\end{array}$ & $\begin{array}{l}\text { Satisfação no Emprego } \\
\text { Poder (Psicologia) } \\
\text { Conflito (Psicologia) }\end{array}$ & $\begin{array}{l}\text { Satisfação no trabalho } \\
\text { Poder } \\
\text { Conflito }\end{array}$ \\
\hline $\begin{array}{l}\text { 3) Reflexões sobre as disputas das } \\
\text { comunidades científicas pela } \\
\text { regulação do campo psi }\end{array}$ & $\begin{array}{l}\text { Psicanálise } \\
\text { Psiquiatria } \\
\text { Psicologia Clínica }\end{array}$ & $\begin{array}{l}\text { Psicanálise clínica } \\
\text { Psiquiatria } \\
\text { Psicologia clínica }\end{array}$ \\
\hline $\begin{array}{l}\text { 4) "Falar com seus botões": pelos } \\
\text { meandros teóricos e empíricos das } \\
\text { relações entre conversa interna, } \\
\text { reflexividade e self }\end{array}$ & $\begin{array}{l}\text { Autoimagem } \\
\text { Psicologia } \\
\text { Desenvolvimento humano } \\
\text { Lógica }\end{array}$ & $\begin{array}{l}\text { Autopercepção } \\
\text { Lógica e psicologia } \\
\text { Psicologia do desenvolvimento }\end{array}$ \\
\hline $\begin{array}{l}\text { 5) Cuidadores de crianças com } \\
\text { leucemia: exigências do tratamento } \\
\text { e aprendizagem de novos } \\
\text { comportamentos }\end{array}$ & $\begin{array}{l}\text { Leucemia } \\
\text { Neoplasias } \\
\text { Cuidadores } \\
\text { Aprendizagem } \\
\text { Terapia comportamental } \\
\text { Psicologia da criança } \\
\end{array}$ & $\begin{array}{l}\text { Leucemia } \\
\text { Neoplasias } \\
\text { Cuidadores } \\
\text { Psico-oncologia pediátrica Aprendizagem } \\
\text { Modificação do comportamento }\end{array}$ \\
\hline
\end{tabular}

Fonte: Dados da pesquisa ${ }^{6}$.

No próximo quadro, verificou-se que 16 artigos apresentaram consistência na indexação realizada, utilizando-se do DeCS, por serem documentos que tratam especificamente da saúde. Por exemplo, o segundo artigo, que fala sobre patologia de linguagem e escuta, recebeu do DeCS o descritor "Patologia da fala e da linguagem", termo específico que representa muito bem o conteúdo, e da Terminologia em Psicologia, "Fonoaudiologia", termo geral que não atingiu a especificidade do documento.

\footnotetext{
${ }^{6}$ Disponível em: <http://newpsi.bvs-psi.org.br/cgi-

bin/wxis1660.exe/iah/?IsisScript=iah/iah.xis\&lang=P\&base=INDEXPSI>. Acesso em: 24 ago. 2012.
} 

analisados.

Esse conteúdo representa aproximadamente $13 \%$ do total dos artigos

Seguem exemplos para facilitar a visualização dos resultados:

Quadro 2 - Indexações mais específicas utilizando o DeCS dos artigos das revistas de psicologia da base LILACS entre os anos 2010-2011

\begin{tabular}{l|l|l}
\hline Título do artigo & Indexação pelo DeCS & $\begin{array}{l}\text { Indexação pela } \\
\text { terminologia em } \\
\text { Psicologia }\end{array}$ \\
\hline \hline $\begin{array}{l}\text { 1) Crenças sobre comportamentos de } \\
\text { saúde e adesão à prevenção e ao } \\
\text { controle de doenças cardiovasculares }\end{array}$ & $\begin{array}{l}\text { Saúde } \\
\text { Doenças Cardiovasculares Doença }\end{array}$ & Distúrbios do coração \\
\hline $\begin{array}{l}\text { 2) Patologia de linguagem e escuta } \\
\text { fonoaudiológica permeada pela } \\
\text { psicanálise }\end{array}$ & $\begin{array}{l}\text { Psicanálise } \\
\text { Fonoaudiologia }\end{array}$ & Linguagem \\
\hline
\end{tabular}

Fonte: Dados da pesquisa ${ }^{7}$.

No seguinte quadro, verificou-se que, por serem da área específica de psicologia, os documentos foram devidamente retratados conceitualmente pela Terminologia em Psicologia.

Quadro 3 - Indexações mais específicas utilizando a Terminologia em Psicologia dos artigos das revistas de psicologia da base LILACS entre os anos 2010-2011

\begin{tabular}{|c|c|c|}
\hline Título do artigo & Indexação pelo DeCS & Indexação pela terminologia em Psicologia \\
\hline $\begin{array}{l}\text { 1) Psicologia comunitária: a clínica ampliada na } \\
\text { localidade de Muzema }\end{array}$ & $\begin{array}{l}\text { Saúde Pública } \\
\text { Psicologia }\end{array}$ & $\begin{array}{l}\text { Saúde Pública } \\
\text { Psicologia comunitária Comunidades }\end{array}$ \\
\hline $\begin{array}{l}\text { 2) Estudo comparativo entre alunos superdotados } \\
\text { e não-superdotados em relações à inteligência e } \\
\text { criatividade }\end{array}$ & $\begin{array}{l}\text { Psicologia } \\
\text { Criatividade } \\
\text { Inteligência }\end{array}$ & $\begin{array}{l}\text { Superdotados } \\
\text { Inteligência } \\
\text { Criatividade } \\
\text { Ensino Fundamental }\end{array}$ \\
\hline $\begin{array}{l}\text { 3) A constituição da maternidade em gestantes } \\
\text { solteiras }\end{array}$ & Gestantes & Gravidez, Maternidade, Mães solteiras \\
\hline $\begin{array}{l}\text { 4) Trabalho de equipe: a teoria na prática para } \\
\text { um gerenciamento eficaz }\end{array}$ & $\begin{array}{l}\text { Organizações } \\
\text { Liderança } \\
\text { Educação Baseada em } \\
\text { competências }\end{array}$ & $\begin{array}{l}\text { Psicologia organizacional } \\
\text { Grupos } \\
\text { Liderança } \\
\text { Competência } \\
\text { Inteligência emocional }\end{array}$ \\
\hline $\begin{array}{l}\text { 5) Cultura, subjetividade e as organizações na } \\
\text { contemporaneidade }\end{array}$ & $\begin{array}{l}\text { Cultura } \\
\text { Organizações } \\
\text { Trabalhadores }\end{array}$ & $\begin{array}{l}\text { Comportamento organizacional } \\
\text { Contemporaneidade } \\
\text { Atitudes do empregado } \\
\text { Subjetividade }\end{array}$ \\
\hline $\begin{array}{l}\text { 6) Superendividamento dos consumidores: uma } \\
\text { abordagem a partir da Psicologia Social }\end{array}$ & $\begin{array}{l}\text { Satisfação dos } \\
\text { consumidores }\end{array}$ & $\begin{array}{l}\text { Finanças, Comportamento do consumidor } \\
\text { Marketing, Psicologia Social }\end{array}$ \\
\hline 7) La subjetivación Del duelo em Freud y Lacan & $\begin{array}{l}\text { Cobertura de Serviços } \\
\text { Públicos de Saúde } \\
\text { Setor Privado } \\
\text { Cobertura de Serviços } \\
\text { Privados de Saúde }\end{array}$ & $\begin{array}{l}\text { Subjetividade } \\
\text { Psicanálise clínica } \\
\text { Objeto (Lacan) }\end{array}$ \\
\hline $\begin{array}{l}\text { 8) Percepção da dinâmica familiar por crianças } \\
\text { antissociais: um estudo comparativo com o } \\
\text { procedimento de desenhos de famílias com } \\
\text { estórias }\end{array}$ & $\begin{array}{l}\text { Psicologia } \\
\text { Criança }\end{array}$ & $\begin{array}{l}\text { Personalidade antissocial } \\
\text { Técnicas projetivas } \\
\text { Psicologia da criança }\end{array}$ \\
\hline 9) A psicoterapia dialógica de Martin Buber & $\begin{array}{l}\text { Psicologia } \\
\text { Psicoterapia }\end{array}$ & $\begin{array}{l}\text { Psicoterapia } \\
\text { Psicólogos } \\
\text { Rogers, Carl Ramson, 1902-1987 } \\
\text { Buber, Martin, 1878-1965 } \\
\text { Terapia Centrada no Cliente }\end{array}$ \\
\hline $\begin{array}{l}\text { 10) Atenção a si: da auto-observação à } \\
\text { autoprodução }\end{array}$ & $\begin{array}{l}\text { Atenção } \\
\text { Cognição } \\
\text { Ciência } \\
\text { Cognitiva }\end{array}$ & $\begin{array}{l}\text { Atenção } \\
\text { Auto-observação } \\
\text { Cognição } \\
\text { Psicologia cognitiva }\end{array}$ \\
\hline
\end{tabular}

Fonte: Dados da pesquisa ${ }^{8}$.

7 Disponível em: <http://newpsi.bvs-psi.org.br/cgi- bin/wxis1660.exe/iah/?IsisScript=iah/iah.xis\&lang=P\&base=INDEXPSI> Acesso em: 24 ago. 2012. 
Em relação à terminologia das Ciências da Saúde, o quinto artigo ilustra a ausência dos termos "Subjetividade" e "Contemporaneidade", muito utilizados no campo da Psicanálise, e que não constam naquele vocabulário.

O sexto título, Superendividamento dos consumidores: uma abordagem a partir da Psicologia Social, apresenta em sua máxima o tema "Comportamento do consumidor", também não contemplado pelo DeCS.

O artigo $A$ constituição da maternidade em gestantes solteiras possibilita uma análise interessante, pois este caso expõe como cada área do conhecimento se comporta diante de um mesmo descritor, no caso, "Maternidade". O DeCS utiliza "Maternidade" para designar o Hospital especializado e para a Terminologia em Psicologia o aspecto é relacionado ao sentido de ser mãe.

Em relação ao artigo La subjetivación del duelo en Freud y Lacan, é possível perceber que houve dificuldade de representá-lo por meio do DeCS. O tema geral é Freud e Lacan e, neste momento, já se pressupõe referir-se à psicanálise. Os termos que foram utilizados para indexar pelo DeCS, contudo, não correspondem ao conteúdo do documento (apesar do termo "Psicanálise" existir nesta terminologia). Já os que foram atribuídos pela Terminologia em Psicologia foram mais exatos em sua representação, por também utilizarem autores como assunto. Isso também ocorre no nono artigo, que apresenta autores como temas principais, o que não pode ser contemplado pela terminologia do DeCS.

Verificou-se, portanto, que os indexadores representaram de modo mais específico e objetivo os 65 artigos utilizando a Terminologia em Psicologia, o que representa $52 \%$ do total dos documentos analisados.

Outro comentário pertinente é sobre o fato de treze artigos terem sido indexados na LILACS apenas com a palavra "Psicologia". Essa situação nos levou à seguinte reflexão: Por que o assunto foi colocado de forma tão geral? Não existe no DeCS um termo mais específico? O indexador desconhece a área a ponto de não identificar e representar o conteúdo do documento de forma mais elaborada?

Vale salientar a importância de uma boa representação temática considerando a satisfação do cliente final e colaborando com o desenvolvimento da psicologia.

Percebeu-se, durante o processo de análise dos dados, que muitos temas não foram adequadamente representados. Ficou evidente em alguns casos que há desconhecimento do assunto tratado, o que gera a não utilização plena das terminologias que possuem suas especificidades.

Citaremos alguns exemplos de termos específicos que apareceram nos artigos analisados e que poderiam ser acrescentados ao DeCS: Objeto (Lacan); Maternidade (sentido da área da psicologia); Alteridade; Subjetividade; Autoconceito; Aprendizagem escolar; Processos cognitivos; Consciência corporal; Psicanálise clínica; Comportamento do consumidor;

\footnotetext{
${ }^{8}$ Disponível em: <http://newpsi.bvs-psi.org.br/cgi-

bin/wxis1660.exe/iah/?IsisScript=iah/iah.xis\&lang=P\&base=INDEXPSI>. Acesso em: 24 ago. 2012.
} 
Psicologia do consumidor; Constituição do sujeito; Psicologia cognitiva; Desenvolvimento emocional; Desenvolvimento cognitivo; Desenvolvimento psicossocial; Psicologia comunitária; Estereótipos (Psicologia); Representação Social; Gozo (Psicanálise); Tristeza; Violência escolar; Violência psicológica; Habilidades sociais; entre outros.

\section{Conclusão}

Os dados apresentados podem nortear uma ação benéfica para auxiliar ambas as áreas no acréscimo de assuntos específicos nas terminologias, atingindo melhores resultados das buscas realizadas na LILACS e atendendo aos usuários finais.

Evidenciou-se que a recuperação e seleção de documentos são mais assertivas, quando se utiliza uma terminologia específica de uma área que a representa, com melhor qualidade e enriquecimento de informação.

Apesar da Terminologia em Psicologia ainda se encontrar em desenvolvimento, ela representa com mais qualidade, no quesito indexação, os artigos desta área específica.

Sugere-se que seja estabelecida uma discussão sobre a importância de serem incorporados ao DeCS alguns descritores mais relevantes da Terminologia da Psicologia, assegurando ao pesquisador a qualidade e a especificidade da informação que ele almeja para o desenvolvimento do seu trabalho e para o progresso da área em questão.

A inclusão beneficiaria o trabalho dos Centros que cooperam com a ReBAP na alimentação, manutenção e atualização da LILACS, pois aumentaria a quantidade de termos da área de Psicologia no DeCS, que, conforme demonstrado por esta pesquisa, atualmente não representa adequadamente a área.

\section{Referências}

ARRIMAR, J. de A. Análise, indexação e recuperação da informação. Rede RBE: Newsletter, n. 6, dez. 2010. Disponível em: <http://www.rbe.minedu.pt/newsletter//np4/869.html>. Acesso em: 21 ago. 2012.

BOCCATO, V. R. C.; FUJITA, M. S. L. Estudo comparativo entre vocabulários controlados de catálogos coletivos em bibliotecas universitárias. In: CONGRESSO BRASILEIRO DE BIBLIOTECONOMIA, DOCUMENTAÇÃO E CIÊNCIA DA INFORMAÇÃO, 24., 2011. Maceió, Alagoas. Anais... Maceió, 2011. Disponível em: <http://febab.org.br/congressos/index.php/cbbd/xxiv/paper/view/43>. Acesso em: 23 ago. 2012.

BORBA, D. S.; VAN DER LAAN, R. H.; CHINI, B. R. Palavras-chave: convergências e diferenciações entre a linguagem natural e a terminologia. Perspectivas em Ciência da Informação, v. 17, n. 2, p. 2636, $2012 . \quad$ Disponível em: <http://portaldeperiodicos.eci.ufmg.br/index.php/pci/article/view/1341>. Acesso em: 23 ago. 2012. 
HISTÓRICO da Lilacs. Disponível em: <http://lilacs.bvsalud.org/>. Acesso em: 2 set. 2012.

HISTÓRICO da Terminologia em Psicologia. Disponível em: $<$ www.psi.homolog.bvs.br/php/level.php?lang=pt\&component=19\&item= 95> Acesso em: 24 ago. 2012.

HISTÓRICO do DeCS Disponível em: <http://decs.bvs.br/P/decsweb2012.htm $\geq$ Acesso em: 24 ago. 2012.

LISTA dos periódicos indexados no Index Psi Revistas Técnico-Científicas. Disponível em: <http://newpsi.bvs-psi.org.br/cgibin/wxis1660.exe/iah/?IsisScript=iah/iah.xis\&lang=P\&base=INDEXPSI > Acesso em: 24 ago. 2012.

LANCASTER, F. W. Indexação e resumos: teoria e prática. 2.ed. rev. e ampl. e atual. Brasília, DF: Briquet de Lemos, 2004.

MANHÃ,$E$. M. et al. Indexação analítica de periódicos da biblioteca da Faculdade de Farmácia da UFMG. In: SEMINÁRIO NACIONAL DE BIBLIOTECAS UNIVERSITÁRIAS, 15., 2008. São Paulo: UNICAMP, 2008. Disponível em: <www.sbu.unicamp.br/snbu2008/anais/site/pdfs/2785.pdf>. Acesso em: 22 ago. 2012.

PACKER, A. Vida longa à BVS-PSI! In: FÓRUM DE ENTIDADES NACIONAIS DA PSICOLOGIA BRASILEIRA - FENPB. BVS-PSI: 10 anos - Divulgando a psicologia. Brasília: CFP, 2011.

SAMPAIO, M. I. C.; SABADINI, A. A. Z. P. La merecida visibilidad de las revistas latinoamericanas de psicología. Revista Colombiana de Psicologia, v. 21, n. 1, p. 111-123, 2012. Disponível em: <http://www.revistas.unal.edu.co/index.php/psicologia/article/viewFile/31 035/31101>. Acesso em: 6 jul. 2012. 